\title{
Understanding Algorithms by Means of Visualized Path Testing*
}

\author{
Ari Korhonen ${ }^{1}$, Erkki Sutinen ${ }^{2}$, and Jorma Tarhio ${ }^{1}$ \\ 1 Department of Computer Science and Engineering \\ Helsinki University of Technology, Finland \\ Helsinki University of Technology \\ P.O. Box 5400, FIN-02015 HUT, Finland \\ \{archie, tarhio\}@cs.hut.fi \\ 2 Department of Computer Science \\ University of Joensuu, Finland \\ sutinen@cs.joensuu.fi
}

\begin{abstract}
.
Visualization of an algorithm offers only a rough picture of operations. Explanations are crucial for deeper understanding, because they help the viewer to associate the visualization with the factual meaning of each detail. We present a framework based on path testing for associating instructive explanations and assignments with a constructive self-study visualization of an algorithm. The algorithm is divided into blocks, and a description is given for each block. The system contains a separate window for code, flowchart, animation, explanations, and control. Assignments are based on the flowchart and on the coverage conditions of path testing. Path testing is expected to lead into more accurate evaluation of learning outcomes because it supports systematic instruction in addition to more free trial-and-error heuristics. A qualitative analysis of preliminary experiences with the prototype indicates that the approach helps a student to reflect on her own reasoning about the algorithm. However, a prerequisite for an successful learning process with the environment is a motivating introduction, describing both the system and the main idea of the algorithm to be learned.
\end{abstract}

\section{Introduction}

A major challenge in Computer Science education is that of learning algorithmic thinking and a related skill, namely programming. While an algorithm is on a more abstract level than a program, they require a similar readiness from a student: she has to be able to comprehend the core, idea, or essence of a computational process, not just its step-by-step running-time behavior with a given input.

Quite often, a hindrance of learning is due to reasoning in a narrow or conventional way. A student concentrates on peripheral issues rather than strives

\footnotetext{
* The work was supported by the National Technology Agency, Finland.
} 
her way into the nucleus of a new topic. In the case of programming, this might lead into an entirely incorrect misconception of a program's key idea.

Computer science educators have tried to solve the problem of learning an algorithm by the help of various visualization tools 4,681117. From a pedagogical perspective, one could divide them into two groups: the ones targeted for instruction and the ones for construction. The first category covers environments consisting of fancy ready-made animations with a possible teacher's voice explaining the algorithm. These animation packages fit well into a teacherdirected learning context. In the second category we have tools, which can be used by the learners themselves for designing their own visualizations; they work for more open-ended learner-centered settings.

In a self-study situation, a student makes use of both instructive and constructive learning tools. To compensate the lack of a teacher, a tool has to mimic a teacher by explaining an algorithm, if possible, on-the-fly. A crucial question is how to provide a student with an environment where she can freely study an algorithm and, at the same time, get instructive feedback on the essential stages of it.

The efficiency of the visual learning tools, whether instructive or constructive, is hard to assess. However, there seems to be a tentative resolution that at least constructive tools help a learner to get a deeper insight into a given algorithm or program. The problem is, however, that in most environments the effort of building an animation takes at least the same time as learning the algorithm in some other way.

Although a constructive animation environment may open the "black box" of a program, its abstract contents might still remain hidden for the visitor. Indeed, the learner will follow how the program runs on a given input, but that is usually not definitely the whole truth. As an example, consider an implementation of the bubble sort working on a sorted sequence.

In the process of understanding the essence of an algorithm or a piece of code, it would, therefore, be attractive to develop technologies which do not only take a learner as a visitor of a black box onto a single ride, corresponding to a program run with one input. Instead, the environment should welcome her as a season-card resident of the box, to get acquainted in the whole box, now as a "glass box". This means that the system should try to help the user to take all the typical paths of the program. This setting is closely related to path testing [2], a widely used software testing technique, where one considers various coverage measures based on paths in the control flow graph of a program.

Naturally, path testing could be used in many ways, and also in conjunction with an instructive or a constructive visualization tool. Basically, a semiautomatic guide could give hints on those paths which the student has not yet discovered. This mechanism could be used within a code editor as well as a visualization tool. Obviously, the bottom-up approach to learning an algorithm by different instances corresponding to different inputs raises questions. For example, what happens if the learner only tries out peripheral or trivial cases, even in a random way? To overcome these kinds of difficulties, a learning environment 
based on path testing should have instructive features. Especially, it should tutor the student to cover all the paths, and after that, following the tradition of Pólya [15], reason about the idea behind all these paths.

Different learners have various kinds of problems in understanding the character of a given program. A promising idea behind the path testing is that each learner can usually start with a path which is simple for her to understand, and then proceed to more demanding paths. Reasoning about the meaning of paths is probably an efficient way to transfer one's understanding above the current zone of proximal development [19]. This learning process can probably take place also within a community of learners, whether physical or virtual.

Intuitively, the idea of path testing comes from tracing potential paths in a flowchart representing a given program. This intuition might mislead one to suspect that the tested paths have to be presented visually on a flowchart. This is, however, not the case. In fact, the idea of path testing is independent of any single visual representation but rather reflects the abstract idea of an algorithm under study. Thus, it can be applied not only in various visualization tools, but virtually in any program concretization environment.

Unlike current technology trends for learning which aim at producing massive but mostly static learning materials for virtual universities, the idea of path testing is based on the need for tools which support learning of skills. Compared to learning knowledge from Web-stored digital libraries, path testing takes learners into an environment where they are supposed to learn and create new ideas by actively identifying novel paths.

The rest of the paper has been organized as follows. We start with introducing some basic concepts of path testing in Section 2. In Section 3 we sketch the guidelines of the learning environment of a new kind for studying algorithms. In Section 4 we describe a pilot environment we made to test our ideas in practice. Before discussing ideas on future development in Section 6, we report results of a qualitative evaluation of our prototype in Section 5 .

\section{Path Testing}

Path testing [25] is a widely-used glass box testing technique, i.e. the source code of a program to be tested is known. A test case corresponds to a certain value combination of input variables. Each test case induces a path in the flow graph of the program. One considers the paths corresponding to a set of test cases under certain coverage conditions.

Test coverage analysis is the process of finding areas of a program not exercised by a test set, i.e. a set of test cases. This involves creating additional test cases to increase coverage and identifying redundant test cases that do not increase coverage. The aim is a complete coverage with a test set of minimal size.

There are several measures of test coverage. The most common ones are the following: 
Statement coverage is satisfied, when every statement is executed at least once with the test set.

Branch coverage is satisfied, when every edge of the flow graph of the program is applied at least once with the test set.

Multiple condition coverage is satisfied, when every possible true/false combination of Boolean sub-expressions of each Boolean expression occurs.

Path coverage is satisfied, when the test set contains a test case for every possible control path in the flow graph of the program.

Clearly path coverage includes branch coverage which in turn satisfies statement coverage. Multiple condition coverage is similar to branch coverage but has better sensitivity to the control flow. However, multiple condition coverage does not necessarily imply branch coverage and path coverage does not guarantee multiple condition coverage in a general case.

Path coverage has the advantage of requiring very thorough testing. Because the number of paths is exponential to the number of branches, full path coverage is seldom useful. In practice weaker measures are used instead of the full path coverage.

As said above, the aim of coverage analysis is to find a test set of minimal size satisfying a certain coverage condition. Because of the difficulty of this optimization problem, one must be satisfied with approximate solutions in practice.

Example. Let us consider a code fragment of two lines with two input variables $\mathrm{a}$ and $\mathrm{b}$ :

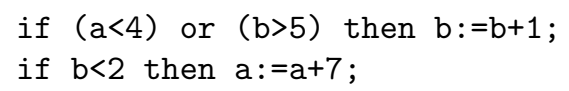

The following test sets of pairs $(a, b)$ satisfy the coverage conditions presented above:

$\begin{array}{ll}\text { Statement coverage: } & \{(0,0)\} \\ \text { Branch coverage: } & \{(0,0),(4,2)\} \\ \text { Multiple condition coverage: } & \{(0,0),(3,6),(4,5),(4,6)\} \\ \text { Path coverage: } & \{(0,0),(0,1),(4,1),(4,2)\}\end{array}$

Note that in this case, multiple condition coverage does not hold for the test set satisfying path coverage and vice versa.

\section{Guidelines of the Approach}

Our aim is to present a new framework of self-study learning environments for algorithms. Our approach framework is partially based on path testing. As a prerequisite, the concepts of path testing should be presented in a self-explaining way so that the learner need not know anything about path testing before using an environment based on our framework. 
The algorithm is divided into blocks. A basic block [1 is a sequence of statements in which flow of control enters at the beginning and leaves at the end without outgoing or incoming branching in the middle. In addition to the linear division to basic blocks, it is beneficial to consider a hierarchical division and apply stepwise refinement 18, because the hierarchy promotes learning of complex algorithms. On the lowest level a block is approximately a basic block. On the highest level there is only one block. Descriptive names could be associated with the blocks.

We use the Boyer-Moore-Horspool (BMH) algorithm [7] for string matching as an example algorithm in order to show how the idea of path testing supports the learning process. String algorithms provide Computer Science educators with a challenge. How to make students - even on an advanced level-understand the distinctive idea of an algorithm which consists only of few lines? A block hierarchy of $\mathrm{BMH}$ is given in Figure 1. Some of the blocks may be left without refinement, like the initialization on the first four lines in Figure 1.

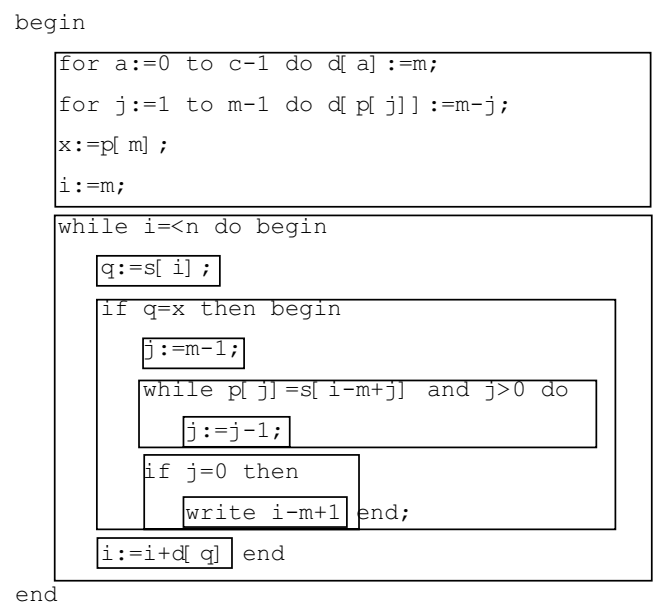

Fig. 1. Block hierarchy of the BMH algorithm.

An explanation describing pre and post conditions and the meaning of actions is given for the blocks. Besides the block descriptions, a general review of the algorithm and explanations of the meanings of the variables are also given. The environment contains a separate window for code, flowchart, animation, explanations, and control. The code window shows the code of the algorithm with the active line highlighted.

The flowchart window is a novel feature. The user may get several views of the execution. The first view shows a single path in the flowchart. Alternatively, the history of execution is shown as a colored worm creeping along the flow lines. The second view shows the activity of flow lines. Applied paths are shown 
such that the width of a line reflects the number of applications. Statement coverage can be checked with this view. The third view is for examining multiple condition coverage. Conditional branch nodes have a mark for every combination of Boolean primaries, which are typically relational expressions. The color of a mark indicates which combinations have occurred.

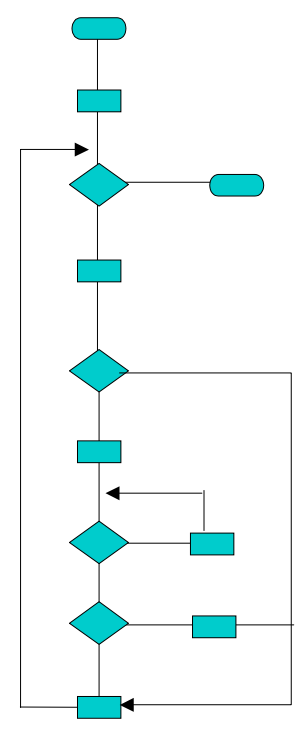

Fig. 2. Flow chart of the BMH algorithm.

The environment provides students with assignments in order to lead them to a more profound understanding and at same time to monitor their learning. Suitable assignments include:

- Give inputs for statement, branch, and multiple condition coverage.

- One of the blocks is left without explanation. Give an explanation.

- Explain how the algorithm proceeds in given situations.

- Some algorithm specific problems.

Because the assignments are prepared in advance, possible problems in the coverage of test sets can be ruled out. The answers consisting of test sets are fairly straightforward to check automatically.

There are several ways the environment can be used. In addition to giving traditional classroom presentation, one can define a homework assignment. In the case of the $\mathrm{BMH}$ algorithm, the student may be asked to define a pattern that satisfies statement coverage. In this example it turns out that statement coverage could be satisfied by only one pattern which occurs in the text. Generally this type of question could be rephrased by asking the student to define a set of 
patterns that satisfies the given condition, i.e., the statement, branch, multiple condition, or path coverage.

Also questions concerning some special features of the algorithm could be asked. In the case of the BMH algorithm, we could ask for a pattern that causes the algorithm "behave badly" (e.g. the algorithm examines each character of the text).

The assignments are formulated in such a way that in many cases a solution may be verified in the environment. After a student thinks that she has solved the given problem, she can simulate the algorithm in the environment, which provides immediate visual feedback of the correctness of the solution.

Questions dealing with explanations are open in the sense that there is not just a single correct solution for them. Therefore it is not possible to provide unique visual feedback about the correctness of a given answer. However, this kind of questions are suitable for classroom discussions.

\section{Prototype}

We implemented a prototype environment to test our ideas in practice. The prototype shows the current values of data structures in a discrete animation of the BMH algorithm. The system has separate code, control and explanation windows. The flowchart window was not implemented, but some of its functions were incorporated with the code window. Instead of a hierarchical block view of the algorithm, code lines are used as blocks.

We chose Matrix [10 12] for the application framework on top of which the prototype was implemented. Matrix is a platform independent visualization and simulation framework that provides extensive set of building blocks for visualizing data structures and algorithms. Even though Matrix is not primarily intended for code animation, but for concept animation and simulation of data structures, it turned out to be suitable for fast prototyping of the new system. The abstraction for visualizing string matching algorithms for this experiment took about 20 hours to complete. Additional 20 hours were used to improve the prototype based on the feedback gathered during testing. Thus, we expect that the creation of similar visualizations for another algorithm will take significantly less time, even if the author were not familiar with the framework. The abstraction for visualizing string matching algorithms has been included in the current version of the Matrix package which is freely available at Matrix homepage [14].

The visualization consists of six separate elements (see a screen snapshot in Figure 3) which are the animator components (buttons labeled Backward, Forward, Begin, End, Play, Reset beginning here, and the slider bar), the array Pattern $P$, the array Shift Table $D$, the two representations of the BMH algorithm (the array representation labeled Text $S$ and the code view representation titled Boyer-Moore-Horspool Algorithm), and finally the optional text window labeled "Description". The user may freely alter the characters in arrays $P$ and $S$. Finally, when ready to execute the algorithm, she just drag \& drops the pattern array into the text array on the screen and the system invokes the 
कै File Edit Options Exercise Help

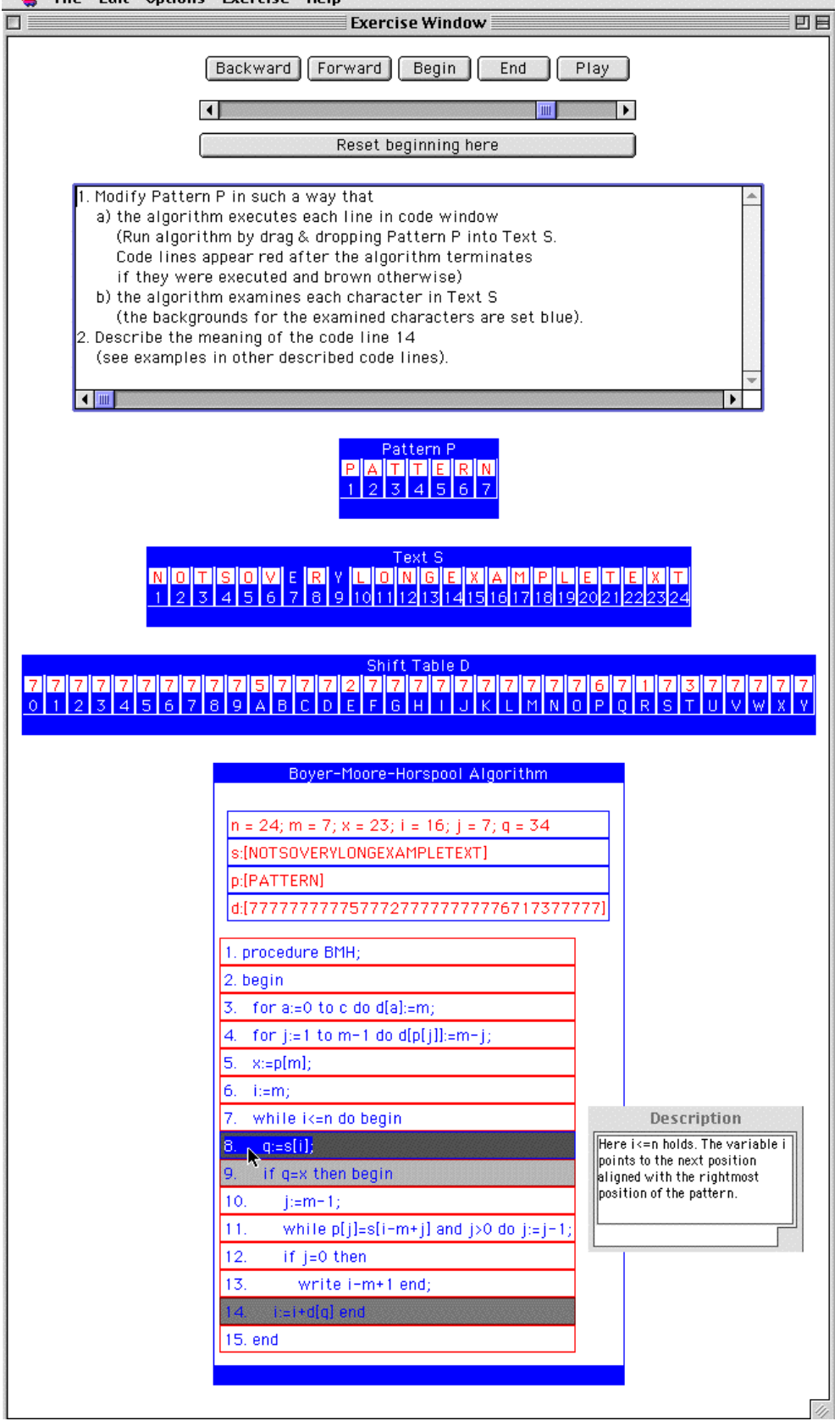

Fig. 3. Screen snapshot of the prototype implementation. 
actual method implementing the $\mathrm{BMH}$ algorithm with the given parameters. Now the visualization sequence appears in the animator and it could be examined through the animator interface. In addition, the execution of the algorithm could be repeated as many times as required with different inputs $P$ and $S$.

\subsection{Creating Code Visualization}

The execution of the algorithm causes the animator to store all the method invocations during the execution in such a way that they could be revoked later on. Thus, the changes to the data structures involved could be restored. This and the issues dealing with data structure visualizations are taken care of the Matrix framework and is not discussed here any further. However, the code visualization is a new feature within Matrix, thus we examine that issue slightly closer.

The actual implementation of the target algorithm $A$ should be written in Java as the Matrix is written in Java. However, the code visualization does not show this actual implementation to the user directly but an abstraction $A^{\prime}$ of it. This abstraction could be written in any language or it could be some kind of pseudo-code presentation or even a plain textual description. In order to make the connection between these two representations of the algorithm, the visualizer should annotate the actual algorithm $A$ by "timestamping" the code lines of the abstraction $A^{\prime}$. We chose this post-mortem technique [16] rather than live visualization because it allows the user to have complete control of the animation (speed, direction, levels of abstractions shown, etc.).

The abstract algorithm $A^{\prime}$ is defined by implementing the interface Code that serves as our abstraction for code visualization. Code includes a set of CodeLines that is another abstraction for a single code line representation. The actual algorithm should be annotated in such a way that the algorithm should invoke CodeLine.stamp (int time) method for each CodeLine just before the actual operation is performed. This assigns a new timestamp for the corresponding code line. Based on this information, Matrix is capable of visualizing the execution of the code as long as the implementation of Code can provide a clock (method int getTime()) that returns the "current time" at any point during the animation.

In addition, the CodeLine interface has a method that could return additional information about the state of the algorithm. Thus, the visualizer can provide a description about the state of the algorithm, the purpose of a single code line, questions concerning the algorithm, etc.

\subsection{Examining of an Algorithm}

To be able to examine the execution of an algorithm $A$ stepwise, the algorithm is first run without visualization. At this point all the lines visited are shown with different color than those lines which were never executed. This was easy to accomplish because those lines never executed still have the timestamp zero and all the other lines have timestamp greater than zero. This approach demonstrates how the statement coverage is illustrated. 
The six animator buttons and the slider bar allow the user to freely traverse through the animation sequence. The five buttons at top of the animator are self-explanatory. The sixth button "Reset beginning here" is used for adjusting the beginning of the animation sequence at given position and thus helps the user to, for example, mark and jump easily to the beginning of the last execution of the algorithm at any time. Moreover, the slider bar could be used to jump at any position in the animation sequence. This kind of interactive algorithm animation helps the user to figure out, for example, why some portions of the code were never executed.

As mentioned above, there are two representations for the BMH algorithm. One illustrates only the text $S$ and the other that includes the algorithm. Because of the nature of Matrix, both of these conceptual views represent the very same physical object that is an instance of the class implementing the string matching abstraction. Thus, there exists only one implementation for the BMH algorithm even though we have two simultaneous representations. Moreover, while the execution of the algorithm makes changes to the shared physical data structures, both representations are updated synchronously. For example, in the upper array representation of the text the changes are expressed by darkening the array position $i$, if it is accessed at line 8 or 11 in the lower code representation.

In the code view the active line of execution is highlighted. Moreover, the lines executed just before the active line are highlighted with lighter shades to illustrate the progress of the program. In addition, an optional text box could be attached to each code line describing the state of the algorithm. In our demonstration this feature is used for describing the pre and post conditions and the meaning of actions for each code block as mentioned in Section 3 .

\section{Experiences}

The prototype was tested with several CS major students, which did not have any prior knowledge about the topic. The first two students gave feedback that was used to improve the prototype before it was introduced to the third student. The final version was fine-tuned based on this latter test case and evaluated by some colleagues. This iterative and incremental design approach gave us the chance to validate and fine-tune the prototype enough to make all the critical design decisions. We discuss the final version shown in Figure 3 and also the improvements made, because the testing process showed that lack of some minor details could have dramatic influence on the learning curve.

The first and expected outcome was that it is not obvious that the students use the system as we expect them to do. For example, the first student tried to completely master the algorithm before starting to watch the visualized simulation. This was somewhat bizarre because we believed that it is the simulation that could be the philosopher's stone to accomplish this task. However, the static code frame is a natural starting point, and the first student spent quite a long time simulating the algorithm in his mind, instead of using our system to do the simulation process. Finally, after the first student managed to solve the 
assignment, he was asked to teach the algorithm to the second one. Even though, the environment forced the first student to use the simulation to complete the assignment, he did not use the simulation capabilities for demonstration purposes while explaining the exercise to the second student. They even used pen and pencil while they discussed the algorithm. Again, the simulation came into the picture after the second student was sure enough of the correctness of his solution and he had the courage to try to solve the assignment.

However, the fact that we should encourage the students to freely explore the algorithm with the system was taken into account when the system was introduced to the third student. A few minutes were spent to illustrate the functionality before the student was let to try it on his own. This time the student started to use the visual debugger at once. He also managed to clarify for himself the logic of the algorithm just by running the algorithm once step by step with the default input. However, also this time the interaction between the user and the system increased during the session. The better he understood the algorithm the more he used the interactive capabilities of the system. The bottom line is that all of the students benefited most from the fact that the system could verify their thinking process and this way give feedback of their performance.

We also noticed that we must provide the student "the big picture" in order to get the learning process started. This is even more important in the case of interactive tools than in the case of traditional methods, because the user cannot predict the amount of material she has to go through. For the time being, our prototype does not include any overall description about the topic or the algorithm, thus the first student really had to struggle before he got some idea of how the algorithm works. Even though normally students are familiar with the topics to be studied, we believe that the overall idea should be provided in order to bring the context in students' mind.

Much of the observed experiences can be interpreted by referring to the individual differences in students' learning styles. Some prefer text-based information to visual one, others find holistic presentation a natural one compared to serial presentation. Basically, a learning environment should adapt to these differences. Adaptive mechanisms are usually rather hard to implement; however, simpler ideas solve the problem at least partly.

For example, texts and other representations can be redundant to some extent. This is because interactive tools, much like regular education, do not represent the whole content at once, but reveal it piece by piece. Thus, for example, the overall idea can be zoomed to highlight further details after the student has discerned the big picture. To quote a Latin proverb, Repetitio mater studiorum est.

We had an option to represent the actual algorithm in Java instead of an abstraction of it. However, representing only an abstraction allows more economic illustrations and hiding of unnecessary details. This leads to better understanding of the logic of an algorithm and reduce problems due to language-specific issues. Moreover, the algorithm may be presented in any language, even in a 
pseudo language. Of course, this approach requires additional work because of the mapping between the actual algorithm and the abstraction of it.

\section{Future Perspectives}

Since our path testing environment is at a stage of an early prototype, there are many alternatives for further development.

First of all, the path testing idea is not at all restricted to our current implementation. It could be incorporated, for example as a tutoring agent, into existing visualization tools. For example Jeliot [36] could be expanded to allow interruptions where a user could assign new values into variables. This way she could study what happens at a loop's end or similar steps of execution. This kind of on-the-fly path testing helps a student to concentrate on the part of the code which she is most interested in.

Path testing can also be used within other kinds of program concretization tools. This is particularly relevant in learning environments targeted at younger learners or K12 programs. For example a flow chart could be easily implemented as a three-dimensional playground where a student could adventure along pipelines. Also robotic learning environments for experimental programming, like Lego/Logo 9] or Empirica Control [13, could get new flavor when a user had to check all potential paths of a robot's behavior.

Since path testing clearly emphasizes the semantics of the program, it could also be applied to a visual proof of the program's correctness. This way the otherwise complicated concepts of semantics could be made more concrete and learned along with introductory programming.

The idea of path testing gives new opportunities to establishing net-based or local learning communities among Computer Science students. Students can reason about their programs in teams, and they get a concrete common space within the realm of a program. Also, exciting competitions can be arranged, like which team is the first one to uncover the secret of a program.

The bottom-up approach of path testing is not only its restriction. Paths discovered and experienced by different students, can serve as crucial steps for a holistic view of the algorithm under study.

Finally, while the system can support the learner by pointing out multiple paths of execution and adjusting the level of detail, it should probably be extended by a particular instructing tool. This tool could open up the interaction by motivating the students to make use of system's capabilities and the whole path testing approach.

\section{References}

1. Aho, A., Sethi, R., Ullman J.: Compilers, Principles, Techniques, and Tools. Addison Wesley, 1986.

2. Beizer, B.: Software Testing Techniques, 2nd edition. New York, Van Nostrand Reinhold, 1990. 
3. Ben-Ari, M., Myller, N., Sutinen, E., Tarhio, J.: Perspectives of Program Animation with Jeliot. In this volume.

4. Brown, M.: Algorithm Animation. ACM distinguished dissertation, MIT Press, 1988.

5. Cornett S.: Code Coverage Analysis. http://www.bullseye.com, seen 08/01.

6. Haajanen, J., Pesonius, M., Sutinen, E., Tarhio, J., Teräsvirta, T., Vanninen, P.: Animation of user algorithms on the Web. In Proc. 1997 Symposium on Visual Languages, 1997, 360-367.

7. Horspool, N.: Practical fast searching in strings. Software Practice \& Experience 10, 1980, 501-506.

8. Hundhausen, C.: Toward Effective Algorithm Visualization Artifacts: Designing for Participation and Communication in an Undergraduate Algorithms Course. Doctoral Dissertation (Tech Rep. No. CIS-TR-99-07), Dept. of Computer and Information Science, University of Oregon, 1999.

9. Järvinen, E. M.: The Lego/Logo Learning Environment in Technology Education: An Experiment in a Finnish Context. Journal of Technology Education 9(2), 1988, $47-59$.

10. Korhonen, A.: Algorithm Animation and Simulation. Licentiate's thesis, Helsinki University of Technology, Department of Computer Science and Engineering, 2000.

11. Korhonen, A., Malmi, L.: Algorithm Simulation with Automatic Assessment. In Proc. ITiCSE '00, 5th Annual SIGCSE/SIGCUE Conference on Innovation and technology in computer science education, ACM, 2000, 160-163.

12. Korhonen, A., Malmi, L., Saikkonen, R.: Design Pattern for Algorithm Animation and Simulation. In E. Sutinen (Ed.): Proceedings of the First Program Visualization Workshop, University of Joensuu, Department of Computer Science, 2001, 89-100.

13. Lavonen, J., Meisalo, V., Lattu, M.: Visual programming: basic structures made easy. In E. Sutinen (Ed.): Proceedings of the First Program Visualization Workshop, University of Joensuu, Department of Computer Science, 2001, 163-177.

14. Matrix homepage: http://www.cs.hut.fi/Research/Matrix/

15. Pólya, G. How to Solve It? Princeton University Press, 1973.

16. Price, B. A., Baecker, R. M., Small, I. S.: A Principled Taxonomy of Software Visualization Journal of Visual Languages and Computing 4(3), 1993, 211-266.

17. Stasko, J., Badre, A., Lewis, C.: Do algorithm animations assist learning: An empirical study and analysis. In Proc. INTERCHI '93, Conference on Human Factors in Computing Systems, Amsterdam, The Netherlands (1993) 61-66.

18. Stern, L., Sondergaard, H., Naish, L.: A strategy for managing content complexity in algorithm animation. In Proc. ITiCSE '99, 4th Annual SIGCSE/SIGCUE Conference on Innovation and technology in computer science education, ACM, 1999, $127-130$.

19. Vygotsky, L. S.: Mind in Society: The Development of Higher Psychological Processes. (M. Cole, V.J. Steiner, S. Scribner, E. Souberman, eds.) Harvard University Press, 1978. 